\title{
Crecimiento y mortalidad del langostino moya Macrobrachium tenellum (Decápoda: Palaemonidae) en el río San Pedro Mezquital, Nayarit, México
}

\author{
Growth and mortality of Macrobrachium tenellum (Decapoda: Palaemonidae) in the San Pedro Mezquital River, Nayarit, Mexico
}

\author{
Manuel Garduño Dionate ${ }^{1}$ Pablo Alejandro Pérez Velázquez², Rosa María Lorán Núñez y y Francisco Rolando Martínez Isunza
}

1 Instituto Nacional de Pesca, Pitágoras 1320, Col. Santa Cruz Atoyac, 03310. CDMX, México ${ }^{2}$ Centro Regional de Investigación Pesquera Bahía de Banderas, Nayarit. Calle Tortuga No. 1, La Cruz de Huanacaxtle, Nayarit, 63732, México ${ }^{3}$ Instituto Nacional de Pesca, Dirección General Adjunta de Investigación Pesquera en el Atlántico. Ejército Mexicano 106. Col. Ex Hacienda YlangYlang. Boca del Río,

Aceptado: 28 de julio de 2017.

Garduño Dionate M., P. A. Pérez Velázquez, R. M. Lorán Núñez y F. R. Martínez Isunza. 2017. Crecimiento y mortalidad del langostino moya Macrobrachium tenellum (Decápoda: Palaemonidae) en el río San Pedro Mezquital, Nayarit, México. Hidrobiológica 27 (2): 359-367. D0l: 10.24275/uam/iz//dcbs/hidro/2017v27n3/Garduno

\section{RESUMEN}

Antecedentes. El aprovechamiento comercial de Macrobrachium tenellum en el río San Pedro Mezquital, Nayarit, inició en 1995 como una pesquería temporal que cubría la demanda local. Posteriormente, la pesquería se intensificó como actividad económica alterna, y logró un impacto regional mediante la producción de alimento y la generación de empleos. Objetivos. Estimar los parámetros de crecimiento y mortalidad de M. tenellum en el río San Pedro Mezquital, Nayarit. Métodos. Los muestreos se realizaron de febrero a octubre de 2010. Para la captura se utilizaron atarrayas de 1.0 y 1.5 pulgadas de luz de malla y un tamaño de muestra de 100 individuos. Los grupos de edad se obtuvieron por el método de Cassie, los parámetros de crecimiento con el método de Walford, la mortalidad total anual por el método de Beverton y Holt y la mortalidad natural con la ecuación de Rikhter y Effanov. Resultados. Las hembras mostraron valores de $\mathrm{L}_{\infty}=$ $19.0 \mathrm{~cm}, \mathrm{P}_{\infty}=55 \mathrm{~g}, \mathrm{k}=0.369 \mathrm{mes}^{-1} \mathrm{y} \mathrm{t}_{0}=-0.035 \mathrm{mes}^{-1}$; en el caso de los machos, fueron $\mathrm{L}_{\infty}=26.6 \mathrm{~cm}, \mathrm{P}_{\infty}=239 \mathrm{~g}, \mathrm{k}=$ $0.221 \mathrm{mes}^{-1} \mathrm{y} \mathrm{t}_{0}=-0.042 \mathrm{mes}^{-1}$. Con base en la relación longitud-peso, las hembras presentaron crecimiento alométrico negativo $(b<3)$ y los machos crecimiento alométrico positivo $(b>3)$. Los parámetros de mortalidad fueron para las hembras $Z=3.84$ año ${ }^{-1}, M=0.72$ año- $^{-1}$ y $E=0.79$ año-1 y para los machos $Z=2.75$ año $0^{-1}, M=0.69$ año $0^{-1}$ y $E=0.70$ año-1. Conclusiones. El langostino Macrobrachium tenellum presenta un ciclo de vida corto menor de dos años, una tasa de crecimiento moderada y tasas de mortalidad total y de explotación altas, lo que ocasiona una sobreexplotación del recurso en el área de estudio.

Palabras clave: Crecimiento alométrico, edad, Macrobrachium tenellum, reclutamiento, tasa de explotación.

\begin{abstract}
Background. Macrobrachium tenellum from the San Pedro Mezquital River in Nayarit, Mexico, started being used in 1995 as a seasonal crustacean that satisfied local demand. Fishing of this species later intensified as an alternative economic activity, with a regional impact based on food production and job creation. Goals. To estimate the growth and mortality parameters of $M$. tenellum from the San Pedro Mezquital River, Nayarit. Methods. Sampling took place from February to October 2010. Drop nets (atarrayas) with mesh sizes of 1 and 1.5 inches were used to collect samples of 100 individuals. Age groups were defined following the Cassie method, growth parameters with the Walford method, total annual mortality with the Beverton and Holt method, and natural mortality with the Rikhter and Effanov equation. Results. Data obtained were for females $L_{\infty}=19 \mathrm{~cm}, P_{\infty}=55 \mathrm{~g}, k=0.369$ month $^{-1}$ and $t_{0}=-0.035$ month $^{-1}$, and for males $L_{\infty}=26.6 \mathrm{~cm}, P_{\infty}=$ $239 \mathrm{~g}, \mathrm{k}=0.221$ month $^{-1}$ and $\mathrm{t}_{0}=-0.042$ month $^{-1}$. Based on the length-weight relationship, the females had negative allometric growth $(b<3)$ and the males positive allometric growth $(b>3)$. The mortality parameters were for females $Z$ $=3.84$ year $^{-1}, \mathrm{M}=0.72$ year $^{-1}$, and $\mathrm{E}=0.79$ year $^{-1}$, and for males $\mathrm{Z}=2.75$ year $^{-1}, \mathrm{M}=0.69$ year $^{-1}$, and $\mathrm{E}=0.70$ year $^{-1}$. Conclusions. The prawn Macrobrachium tenellum has a short life cycle of less than two years, a moderate growth rate, and high rates of total mortality and exploitation, which result in an over-exploitation of the resource in the study area.
\end{abstract}

Key words: Age, allometric, exploitation rate, Macrobrachium tenellum, recruitment. 


\section{INTRODUCCIÓN}

Macrobrachium tenellum (Smith, 1871), conocido como camarón moya, se captura y comercializa en la cuenca baja del río San Pedro Mezquital, México. La especie se distribuye desde Mulegé, Baja California Sur, en la parte media y oriental de la península, hasta su extremo sur y desde Yavaros, Sinaloa, en México, hasta el río Chira, en Perú e isla Galápagos, en Ecuador (Holthuis, 1952b; Boschi, 1974; Guzmán et al., 1981; New, 1988; Ruiz-Santos, 1988). Habita en aguas dulces, salobres y lagunas costeras (Boschi, 1974) y en todo tipo de fondos (Boschi, 1974; Román-Contreras, 1991; Hendrickx, 1995; Signoret-Poillon \& Soto, 1997; Arroyo-Rentería \& Magaña-Ríos, 2001; Espino-Barr et al., 2006).

El aprovechamiento comercial del camarón moya en el río San Pedro Mezquital inició en 1995 como una pesquería temporal que cubría la demanda local. Posteriormente, la pesquería se intensificó como una actividad económica alterna con un impacto regional mediante la producción de alimento y la generación de empleos temporales directos e indirectos, principalmente en la temporada de veda de otros recursos pesqueros e incluso durante el periodo de veda de esta especie, del 1 de agosto al 31 de octubre de cada año (DOF, 1994). En Nayarit, el camarón moya se captura principalmente en la temporada de lluvias de junio a octubre, debido a su migración y ciclo de vida, observándose una alta proporción de machos y hembras adultos, en su mayoría hembras ovígeras, en las capturas (Pérez-Velázquez et al., 2011).

Aunque la fisiología de los crustáceos es muy diferente a la de los peces, su crecimiento, en la mayoría, se adecúa al modelo de crecimiento de von Bertalanffy (Musick \& Frazer, 1999). Por ello, las estimaciones de los parámetros de crecimiento y de las tasas de mortalidad y explotación son parte importante del estudio de la biología de los crustáceos de interés comercial, ya que permiten determinar las características biológicas de la especie y el nivel de explotación de la población. A su vez, estos datos proporcionan elementos científicos para la ejecución de políticas de administración y regulación de los recursos pesqueros que permitan un adecuado aprovechamiento. El objetivo de este trabajo fue determinar la estructura de las longitudes y los parámetros de crecimiento, mortalidad y explotación de la población de $M$. tenellum en las zonas de captura de Mexcaltitán, San Miguel Aztlán y Boca de Camichín del río San Pedro Mezquital, Nayarit, México.

\section{MATERIALES Y MÉTODOS}

El río San Pedro Mezquital tiene una longitud de $255 \mathrm{~km}$ y drena una región aproximada de 26,480 km². Hacia el final de su recorrido se bifurca, formando el estero de Las Corrientes, la laguna de Agua Dulce y la laguna de Mexcaltitán, lo que representa una gran extensión de esteros y canales unidos con las aguas del estuario Boca de Camichín en su salida al mar. El área de estudio incluyó tres sitios de captura en el río San Pedro Mezquital: San Miguel Aztlán $\left(21^{\circ} 49^{\prime} 20.55^{\prime \prime} \mathrm{N}\right.$, $105^{\circ} 23^{\prime} 40.40^{\prime \prime}$ ), isla de Mexcaltitán (215' $13.24^{\prime \prime} \mathrm{N}, 105^{\circ} 28^{\prime} 35.15^{\prime \prime}$ 0) y Boca de Camichín (2144'32.92” N, 105²9'30.30” 0) (Fig. 1).

En cada sitio se realizaron muestreos mensuales de febrero a octubre de 2010 durante el cuarto creciente 0 cuarto menguante de la fase lunar, de 23:00 a 04:00 horas del día siguiente en bajamar. El método de pesca utilizado fue la atarraya, con luz de malla de 1.0 y 1.5 pulgadas (2.54 y $3.81 \mathrm{~cm}$, respectivamente). La captura total fue de 1,280 machos y 1,772 hembras de camarón moya, pero se trabajó con una muestra mensual aleatoria de 100 individuos procedentes de los tres sitios de captura, repartidos de manera equitativa, para procesar un total de 900 individuos para el estudio del crecimiento y la mortalidad de M. tenellum. La captura se transportó en bolsas de plástico etiquetadas y con hielo molido para su posterior revisión en el laboratorio húmedo del Centro Regional de Investigación Pesquera Bahía de Banderas, Nayarit. Se registraron las variables de longitud total (LT), tomada desde el extremo apical del rostro hasta la punta del telson con un vernier con precisión de $0.1 \mathrm{~mm}$, y el peso total (PT), tomado con una balanza analítica con precisión de $0.1 \mathrm{~g}$ (Román-Contreras, 1979). En los estudios de edad y crecimiento de crustáceos se utiliza regularmente el análisis de frecuencia de tallas; en este estudio se utilizó el método de Cassie (1954) para estimar los grupos de edad que integran la población de M. tenellum de los tres sitios de captura. Una vez estimada la longitud total promedio de los grupos de edad que integran la población, se aplicó el método de Walford (1946) para estimar la longitud asintótica teórica $\left(\mathrm{L}_{\infty}\right)$ y la tasa de crecimiento de la población (k). Para obtener t se utilizó la función logarítmica inversa de la ecuación de von Bertalanffy (Sparre \& Venema, 1992) y la talla de eclosión de M. tenellum de $1.7 \mathrm{~cm}$ (primera zoea), la cual fue estimada por Meruane et al. (2006). Su valor fue incorporado en los cálculos de la ecuación de crecimiento en longitud y peso.

Para comparar el desempeño del crecimiento de $M$. tenellum, se calculó el índice de crecimiento estándar (phi prima ø’) con las estimaciones previas de la longitud asintótica teórica y la tasa de crecimiento con la siguiente ecuación:

$$
\emptyset^{\prime}=\log _{10}(k)+2 \log _{10}\left(L_{\infty}\right) \text { (Pauly \& Munro, 1984). }
$$

El peso asintótico se estimó mediante la fórmula citada por Csirke (1980), $P_{\infty}=a^{*}\left(L_{\infty}\right)^{b}$, donde $P_{\infty}$ es el peso asintótico y a es el valor de la ordenada al origen de la regresión lineal de la relación longitud-peso. Se establecieron las relaciones longitud-peso de machos, de hembras y de ambos sexos por medio de la ecuación potencial: $P=a^{*}(L)^{b}$, donde $a$ y $b$ son los parámetros de la ecuación calculada mediante regresión lineal simple, previa transformación logarítmica. Se evaluó estadísticamente el valor del coeficiente de regresión $(b)$ por medio de la prueba $t$ de Student (Zar, 1999), para determinar el tipo de crecimiento de la especie. Si $b=3$, el crecimiento se consideró isométrico, y si $b \neq 3$ el crecimiento se consideró alométrico (Ricker, 1975).

La mortalidad total anual (Z) se estimó mediante la ecuación propuesta por Beverton y Holt (1957), utilizando la longitud media de la captura (LM), la longitud de explotación (L') y los parámetros de crecimiento $L_{\infty}$ y $k$; la ecuación para la mortalidad anual fue $Z=k^{*}\left(L_{\infty}-L M\right)$ / LM - L'. La mortalidad natural se determinó por el método de Rikhter y Effanov (1976), el cual se basa en el supuesto de que los peces con una mortalidad natural elevada maduran precozmente y de que existe una relación entre la mortalidad natural $(\mathrm{M})$ y la edad de primera madurez (Epm), usando la ecuación $\mathrm{M}=1.521$ / (Epm $\left.{ }^{0.720}\right)-0.155$. Para evaluar el estatus de la población, se calculó la tasa de explotación anual $(\mathrm{E})$ con la ecuación $E=F / Z$ * $\left(1-\exp ^{(-Z)}\right)$; la mortalidad por pesca $(F)$ se obtuvo calculando la diferencia entre la mortalidad total y mortalidad natural, con la fórmula $\mathrm{F}=\mathrm{Z}-\mathrm{M}$. 


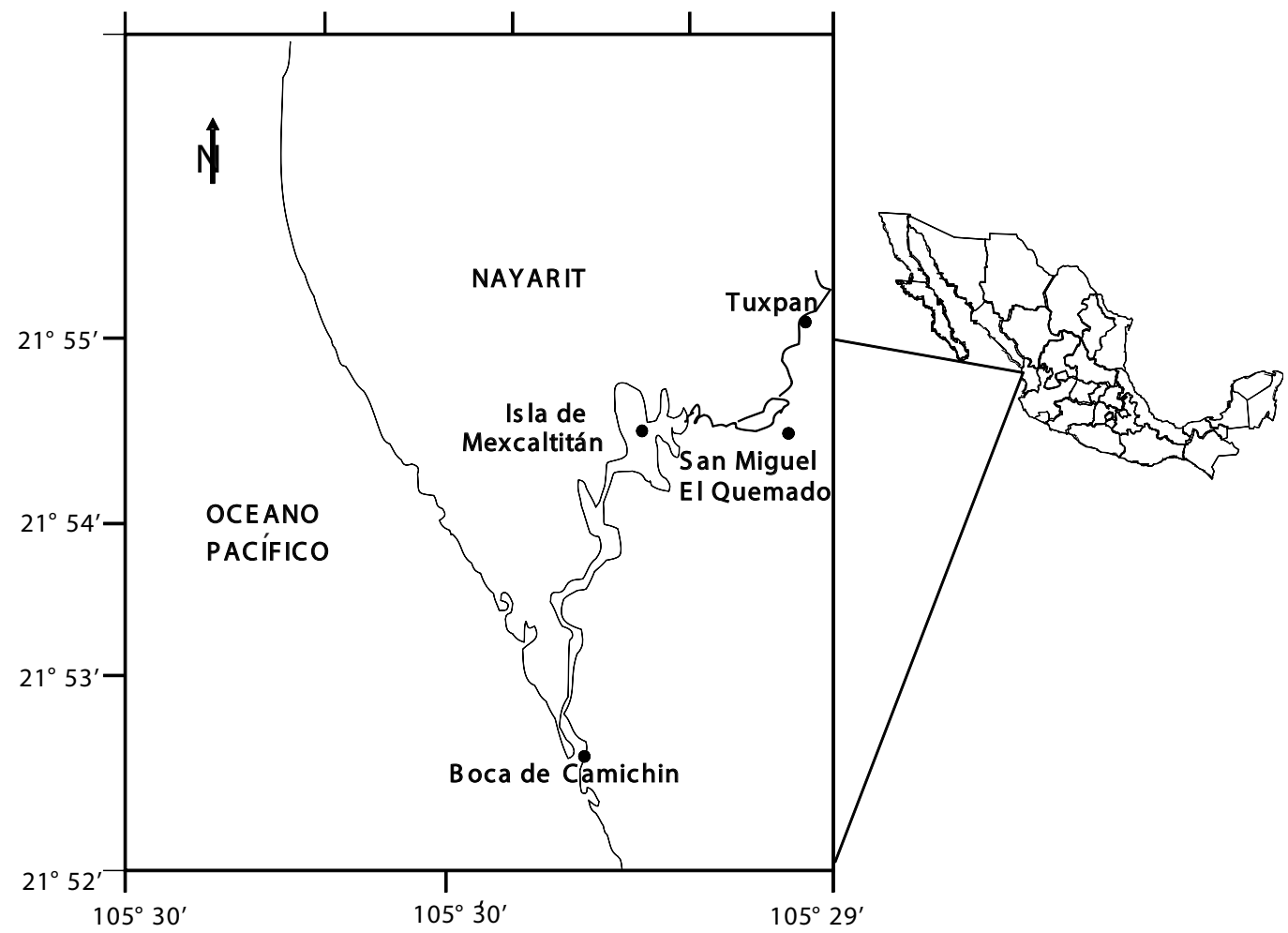

Figura 1. Sitios de captura de Macrobrachium tenellum.

\section{RESULTADOS}

En los machos, la longitud y el peso promedio presentaron una variabilidad de $7.6-13.6 \mathrm{~cm}$ y $3.9-27.3 \mathrm{~g}$, respectivamente, con individuos de menor talla $(7.6 \mathrm{~cm})$ y peso $(3.9 \mathrm{~g})$ promedio en el mes de febrero y de mayor talla $(13.6 \mathrm{~cm})$ y peso $(27.3 \mathrm{~g})$ promedio en septiembre. En las hembras, la longitud y el peso promedio fueron $6.8-10.7 \mathrm{~cm}$ y $3.2-11.4 \mathrm{~g}$, respectivamente, con una menor talla $(6.8 \mathrm{~cm})$ y peso $(3.2 \mathrm{~g})$ promedio en el mes de abril y una mayor talla $(10.7 \mathrm{~cm})$ y peso $(11.4 \mathrm{~g})$ promedio en septiembre. En machos, la mayor captura fue de $14,665 \mathrm{~g}$ en el mes de septiembre; para las hembras, de 5,164 g en agosto (Tabla 1). En la Figura 2 se muestra la distribución mensual de la longitud total en machos y hembras. En febrero, la fracción poblacional de machos y hembras presentó una distribución de tipo polimodal integrada por cohortes de individuos de diferentes tallas, con una longitud y peso totales promedio de $7.6 \mathrm{~cm}$ y $3.9 \mathrm{~g}$ para machos y $8.0 \mathrm{~cm}$ y 4.7 $\mathrm{g}$ para hembras. En marzo, la distribución de las tallas fue de tipo polimodal para machos y modal para las hembras. En este mes se inició la diferenciación de tamaños promedio entre los individuos, que fue para machos de $9.4 \mathrm{~cm}$ y $7.6 \mathrm{~g}$ y para hembras de $7.6 \mathrm{~cm}$ y $3.8 \mathrm{~g}$. En abril, la distribución de tallas de la población fue de tipo polimodal, con machos de mayor talla $(9.3 \mathrm{~cm})$ y peso $(7.9 \mathrm{~g})$ promedio que las hembras $(6.8$ $\mathrm{cm}$ y $3.2 \mathrm{~g}$, respectivamente). En mayo, la distribución fue polimodal en machos y hembras, sobresaliendo la diferenciación de tamaños entre

Tabla 1. Longitud total promedio, peso total promedio y captura mensual de machos y hembras de Macrobrachium tenellum en el río San Pedro Mezquital, Nayarit.

\begin{tabular}{|c|c|c|c|c|c|c|}
\hline \multirow[t]{2}{*}{ Mes } & \multicolumn{3}{|c|}{ Machos } & \multicolumn{3}{|c|}{ Hembras } \\
\hline & $\begin{array}{l}\text { Longitud (cm) } \\
\qquad(\mathrm{P} \pm \mathrm{DE})\end{array}$ & $\begin{array}{c}\text { Peso (g) } \\
(P \pm D E)\end{array}$ & Captura (g) & $\begin{array}{l}\text { Longitud (cm) } \\
\qquad(\mathrm{P} \pm \mathrm{DE})\end{array}$ & $\begin{array}{c}\text { Peso }(g) \\
(P \pm D E)\end{array}$ & Captura (g) \\
\hline febrero & $7.6 \pm 1.282$ & $3.9 \pm 2.165$ & 304 & $8.0 \pm 1.613$ & $4.7 \pm 2.990$ & 108 \\
\hline marzo & $9.4 \pm 1.311$ & $7.6 \pm 3.278$ & 323 & $7.6 \pm 1.146$ & $3.8 \pm 1.806$ & 213 \\
\hline abril & $9.3 \pm 1.645$ & $7.9 \pm 4.158$ & 254 & $6.8 \pm 1.451$ & $3.2 \pm 1.802$ & 313 \\
\hline mayo & $11.2 \pm 1.549$ & $12.6 \pm 4.560$ & 659 & $8.0 \pm 1.500$ & $5.1 \pm 2.709$ & 1078 \\
\hline junio & $10.7 \pm 1.691$ & $13.6 \pm 5.474$ & 1311 & $8.2 \pm 1.111$ & $5.8 \pm 2.655$ & 1078 \\
\hline julio & $10.4 \pm 1.107$ & $13.1 \pm 3.933$ & 514 & $8.3 \pm 0.595$ & $5.4 \pm 1.231$ & 294 \\
\hline agosto & $12.2 \pm 1.195$ & $21.6 \pm 6.395$ & 8622 & $10.1 \pm 0.864$ & $9.6 \pm 2.485$ & 5164 \\
\hline septiembre & $13.6 \pm 1.248$ & $27.3 \pm 7.012$ & 14665 & $10.7 \pm 1.182$ & $11.4 \pm 3.393$ & 4867 \\
\hline octubre & $12.2 \pm 1.534$ & $22.4 \pm 10.023$ & 629 & $9.8 \pm 0.892$ & $9.5 \pm 2.618$ & 1836 \\
\hline
\end{tabular}

$\mathrm{P}=$ promedio; $\mathrm{DE}=$ desviación estándar. 
ambos sexos, con machos de talla promedio de $11.2 \mathrm{~cm}$ y peso promedio de $12.6 \mathrm{~g}$ y la captura de hembras de menor talla $(8.0 \mathrm{~cm})$ y peso $(5.1 \mathrm{~g})$ promedio. En junio, ambos sexos presentaron una distribución polimodal de tallas, con machos de mayor talla $(10.7 \mathrm{~cm})$ y peso $(13.6$ g) promedio que las hembras $(8.2 \mathrm{~cm}$ y $5.8 \mathrm{~g}$, respectivamente). En julio, la distribución de tallas fue de tipo polimodal para los machos y modal para las hembras, con una captura moderada. En agosto, la distribución de tallas fue de tipo modal para los machos y las hembras, con machos de mayor longitud y peso promedio, en comparación con las hembras. En este mes se dio una importante captura $(8,622 \mathrm{~g})$ de machos, integrada por individuos con una longitud y un peso promedio de $12.2 \mathrm{~cm}$ y $21.6 \mathrm{~g}$, respectivamente. También se capturaron 5,164 $\mathrm{g}$ de hembras, con una longitud y un peso promedio de $10.1 \mathrm{~cm}$ y 9.6 $\mathrm{g}$, respectivamente. En septiembre, la distribución de tallas fue de tipo modal para ambos sexos; se capturaron machos de talla promedio de $13.6 \mathrm{~cm}$ y peso promedio de $27.3 \mathrm{~g}$. Finalmente, en octubre, la distribución de tallas fue polimodal para los machos y modal para las hembras y se redujeron las capturas de manera importante con respecto al mes anterior (629 g de machos y 1,836 $\mathrm{g}$ de hembras).

Tabla 2. Parámetros de crecimiento en machos, hembras y ambos sexos de Macrobrachium tenellum y valores de la relación longitudpeso en el río San Pedro Mezquital, Nayarit.

\begin{tabular}{lccc}
\hline Parámetro & Machos & Hembras & Población \\
\hline $\mathrm{L}_{\infty}(\mathrm{cm})$ & 26.6 & 19 & 23.5 \\
$\mathrm{P}_{\infty}(\mathrm{g})$ & 239 & 55.3 & 142 \\
$\mathrm{k}\left(\mathrm{mes}^{-1}\right)$ & 0.221 & 0.369 & 0.232 \\
$\mathrm{t}_{0}\left(\mathrm{mes}^{-1}\right)$ & -0.028 & -0.023 & -0.030 \\
$\mathrm{a}$ & 0.005 & 0.013 & 0.006 \\
$\mathrm{~b}$ & 3.253 & 2.835 & 3.163 \\
\hline
\end{tabular}

$\mathrm{L}_{\infty}=$ Longitud infinita; $\mathrm{P}_{\infty}=$ peso infinito; $\mathrm{k}=$ tasa de crecimiento; $\mathrm{t}_{0}=$ tiempo de inició del crecimiento; $\mathrm{a}=$ intersección ordenada de origen de la relación longitud-peso; $b=$ coeficiente de isometría.
Los parámetros de crecimiento estimados de $\mathrm{L}_{\infty}, \mathrm{k}$ y $\mathrm{t}_{0}$ para hembras, machos y la población total de $M$. tenellum se muestran en la Tabla 2. Éstos se utilizaron para elaborar las curvas de crecimiento en longitud y peso respectivas (Figs. 3 y 4), previa evaluación del valor de la intersección con las ordenadas (a) y de la pendiente de la curva $(b)$ de la regresión lineal en la relación longitud-peso.

La relación longitud-peso de las hembras, machos y población se presenta en la Figura 5 con valores del exponente de $b=2.8357$ (hembras), $b=3.2538$ (machos) y $b=3.1635$ (población). Estos exponentes indicaron un crecimiento alométrico negativo para las hembras y un crecimiento alométrico positivo para los machos y la población. Estos tipos de crecimiento presentaron diferencias estadísticas significativas, como lo indica la prueba $t$ de Student $(p<0.05)$ : $t=0.0015$ (hembras), $\mathrm{t}=0.0120$ (machos) y $\mathrm{t}=0.0066$ (población).

En la Tabla 3 se muestra la comparación de los parámetros de crecimiento estimados en este estudio con los de otros autores. Se observaron diferencias importantes, principalmente en el valor del tiempo cero, y de manera secundaria en la longitud asintótica y la tasa de crecimiento. En la Tabla 4 se presentan los valores de las tasas de mortalidad por pesca y de mortalidad total anual, las cuales fueron altas, principalmente para las hembras. La tasa de explotación para los machos fue de 0.70 y para las hembras de 0.79 , lo que indica que en los tres sitios de captura la actividad pesquera tiene un impacto importante sobre el recurso.

\section{DISCUSIÓN}

Uno de los problemas para determinar la edad de los crustáceos es la ausencia de estructuras calcificadas que contengan los anillos de crecimiento, por lo que para este fin existe el análisis de frecuencia de tallas, con adecuaciones a la función de crecimiento de von Bertalanffy. En este trabajo, mediante la integración de la talla de eclosión de $M$. tenellum en su primera fase larval de zoea, se logró un mejor ajuste de la ecuación de crecimiento en talla y peso, lo que mejora los resultados disponibles para esta especie.

Tabla 3. Parámetros de crecimiento de Macrobrachium tenellum estimados por diferentes autores.

\begin{tabular}{|c|c|c|c|c|c|c|}
\hline Referencia & Localidad & $\begin{array}{l}\mathrm{L}_{\infty} \\
(\mathrm{cm})\end{array}$ & $\begin{array}{c}\mathrm{k} \\
\left(\mathrm{mes}^{-1}\right)\end{array}$ & $\begin{array}{c}t_{0} \\
\left(\mathrm{mes}^{-1}\right)\end{array}$ & $\phi^{\prime}$ & Método \\
\hline \multirow{2}{*}{$\begin{array}{l}\text { Guzmán-Arroyo } \\
\text { (1987) }\end{array}$} & \multirow{2}{*}{$\begin{array}{l}\text { Lagunas Mitla, Coyuca, } \\
\text { Tres Palos y Chautengo, } \\
\text { Gro. }\end{array}$} & $15.4(\mathrm{M})$ & $0.205(\mathrm{M})$ & $-0.517(\mathrm{M})$ & $\phi^{\prime}=1.68(\mathrm{M})$ & \multirow{2}{*}{$\begin{array}{l}\text { Walford (1946), Pauly \& } \\
\text { Munro (1984) }\end{array}$} \\
\hline & & $10.6(\mathrm{H})$ & $0.209(\mathrm{H})$ & $-1.332(\mathrm{H})$ & $\phi^{\prime}=1.37(\mathrm{H})$ & \\
\hline \multirow{2}{*}{$\begin{array}{l}\text { Ruiz-Santos } \\
(1988)\end{array}$} & \multirow[t]{2}{*}{ Laguna Tres Palos, Gro. } & $14.2(\mathrm{M})$ & $0.303(\mathrm{M})$ & $-0.417(\mathrm{M})$ & $\phi^{\prime}=1.78(\mathrm{M})$ & \multirow{2}{*}{$\begin{array}{l}\text { Walford (1946), Pauly \& } \\
\text { Munro (1984) }\end{array}$} \\
\hline & & $11.5(\mathrm{H})$ & $0.223(\mathrm{H})$ & $-0.015(\mathrm{H})$ & $\phi^{\prime}=1.46(H)$ & \\
\hline $\begin{array}{l}\text { Espino-Barr } \\
\text { et al. (2006) }\end{array}$ & Ríos Armería y Naranjo, Col. & 17.9 & 0.543 & -0.020 & $\phi^{\prime}=2.24(\mathrm{P})$ & $\begin{array}{l}\text { Elephant, en Sparre \& } \\
\text { Venema (1992), Pauly \& } \\
\text { Munro (1984) }\end{array}$ \\
\hline \multirow{3}{*}{$\begin{array}{l}\text { El presente } \\
\text { estudio }\end{array}$} & \multirow{3}{*}{$\begin{array}{l}\text { San Miguel Aztlán, Isla } \\
\text { Mexcaltitán y Boca } \\
\text { Camichín, Nay. }\end{array}$} & $26.6(\mathrm{M})$ & $0.221(\mathrm{M})$ & $-0.042(\mathrm{M})$ & $\phi^{\prime}=2.19(\mathrm{M})$ & \multirow{3}{*}{$\begin{array}{l}\text { Cassie (1954), Walford } \\
\text { (1946), Sparre \& } \\
\text { Venema 1992), Pauly \& } \\
\text { Munro (1984) }\end{array}$} \\
\hline & & $19.0(\mathrm{H})$ & $0.369(\mathrm{H})$ & $-0.035(\mathrm{H})$ & $\phi^{\prime}=2.12(\mathrm{H})$ & \\
\hline & & & & & $\phi^{\prime}=2.10(\mathrm{P})$ & \\
\hline
\end{tabular}

$\mathrm{L}_{\infty}=$ longitud infinita; $\mathrm{k}=$ tasa de crecimiento; $\mathrm{t}_{0}=$ tiempo de inicio del crecimiento; $\phi^{\prime}=$ índice de crecimiento estándar. 


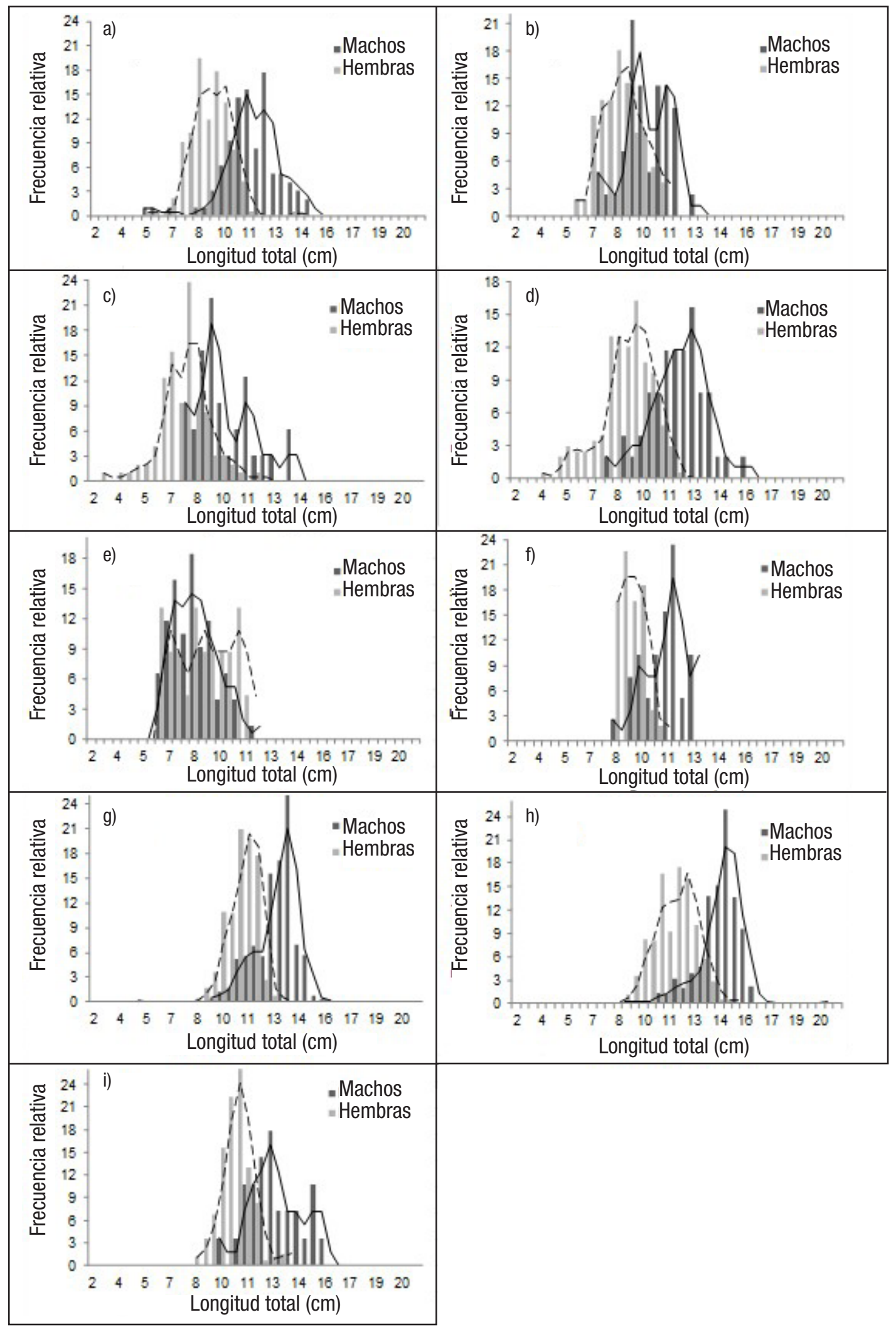

Figuras 2a-i. Estructura de tallas por mes del langostino Macrobrachium tenellum. Las mediciones se realizaron durante nueve meses consecutivos en 2010: a) febrero, b) marzo, c) abril, d) mayo, e) junio, f) julio, g) agosto, h) septiembre, i) octubre. 


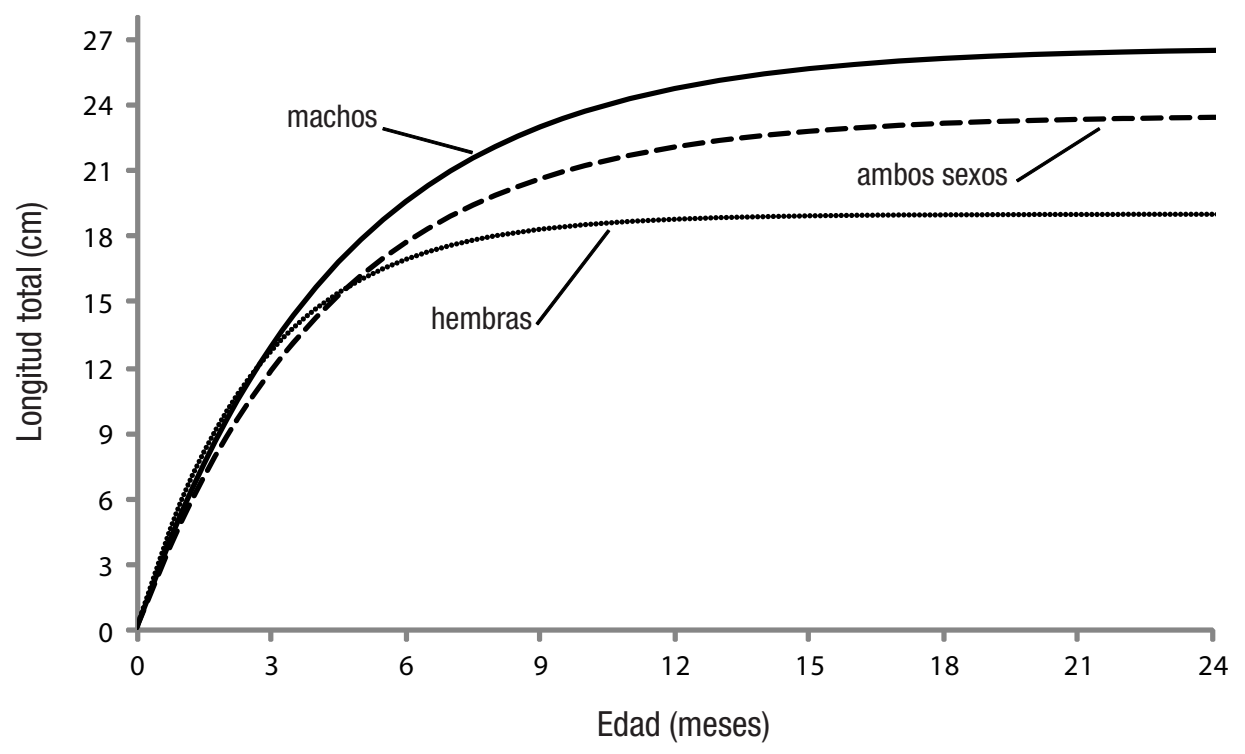

Figura 3. Curvas de crecimiento en longitud de la población y de cada sexo de Macrobrachium tenellum.

Las mayores capturas de camarón moya ocurrieron en agosto y septiembre; estas capturas estuvieron integradas principalmente por hembras maduras y ovígeras, así como por machos de tallas grandes. En estas capturas se observaron concentraciones de individuos en zonas cercanas a la desembocadura del río San Pedro Mezquital, que es donde se produce la eclosión de los huevos (Bahamonde \& Vila, 1971; Norambuena, 1977). Relacionando el aspecto reproductivo con el pesquero, se observó que la actividad pesquera de este recurso se incrementó en la época de lluvias (de julio a octubre) (Ruiz-Santos, 1988; Hendrickx, 1995; Arroyo-Rentería \& Magaña-Ríos, 2001). La pesca de $M$. tenellum se realiza en ríos, esteros y cuerpos de agua costeros de manera intensa y es común en muchas comunidades rurales de la costa del Pacífico (Hernández-Sandoval, 2008); representa un recurso pesquero importante y altamente explotado por las comunidades locales (Cabrera-Jiménez et al., 1977). En este estudio, los machos de camarón moya alcanzaron un mayor tamaño que las hembras, posiblemente debido a un menor valor en la tasa de crecimiento, la cual fue indirectamente proporcional a la longitud asintótica teórica que presentaron.

Esta diferencia en el crecimiento entre sexos fue evidente en el mayor desarrollo de las quelas en los machos, característica descrita para el género Macrobrachium (Holthuis, 1952b; Mossolin \& Bueno, 2003; Mariappan \& Balasundaram, 2004; Hernández et al., 2007). El mayor desarrollo de la quela en machos adultos es una característica

Tabla 4. Tasas de mortalidad y explotación de hembras, machos y ambos sexos de Macrobrachium tenellum en el río San Pedro Mezquital, Nayarit.

\begin{tabular}{cccc}
\hline Parámetros & Machos & Hembras & Población \\
\hline M & 0.69 & 0.72 & 0.62 \\
F & 2.06 & 3.12 & 2.91 \\
Z & 2.75 & 3.84 & 3.54 \\
E & 0.70 & 0.79 & 0.80 \\
\hline
\end{tabular}

$\mathrm{M}=$ mortalidad natural; $\mathrm{F}=$ mortalidad por pesca; $\mathrm{Z}=$ mortalidad total anual; $\mathrm{E}$ $=$ tasa de explotación anual. adaptativa desarrollada a nivel interespecífico e intraespecífico para la defensa, el combate, la alimentación y la reproducción, siendo en este último caso importante para la protección de la hembra durante el apareamiento (Mossolin \& Bueno, 2003; Mariappan \& Balasundaram, 2004). En las hembras la menor longitud asintótica teórica es congruente con el valor más alto de la tasa de crecimiento y puede explicarse por el mayor gasto energético que las hembras experimentan durante el proceso reproductivo, limitando su crecimiento (Mossolin \& Bueno, 2003; Fransozo et al., 2004). Los parámetros de crecimiento de longitud asintótica y tasa de crecimiento de este trabajo fueron mayores a los estimados por otros autores en otras localidades pesqueras (Guzmán-Arroyo, 1987; Ruiz-Santos, 1988; Espino-Barr et al., 2006). Esto puede deberse a diferencias en las condiciones ambientales de las localidades de captura, al tamaño de malla usada para la captura, al tamaño de los individuos en los muestreos, al método de evaluación del crecimiento y al nivel de explotación de la población.

En el presente estudio se integró a la ecuación de crecimiento de von Bertalanffy la talla de eclosión de M. tenellum (Lo) de $1.7 \mathrm{~mm}$, lo que permitió obtener un valor más cercano al teórico esperado (cero) tanto en machos como en hembras. Por esta razón los tiempos cero en este trabajo fueron menores a los estimados por otros autores, lo que mejoró el ajuste de las curvas de crecimiento de longitud y peso para la especie. La evaluación del índice de crecimiento estándar presentó valores similares entre la población de $M$. tenellum de las localidades de Mexcaltitlán, San Miguel Aztlán y Boca de Camichín, Nayarit y las poblaciones de los ríos Armería y Naranjo en Colima (Tabla 3), lo que indica que la especie presenta un similar patrón de crecimiento (AlejoPlata et al., 2011). Sin embargo, el patrón de crecimiento de camarón moya en los sitios aquí estudiados fue diferente del reportado para localidades del estado de Guerrero (Tabla 3), posiblemente debido a la diferencia en los parámetros de crecimiento de longitud asintótica y tasa de crecimiento.

En este trabajo se obtuvieron valores altos de mortalidad total anual, mortalidad por pesca y tasa de explotación para machos y hembras de camarón moya. Estos altos valores pueden deberse a diversas 


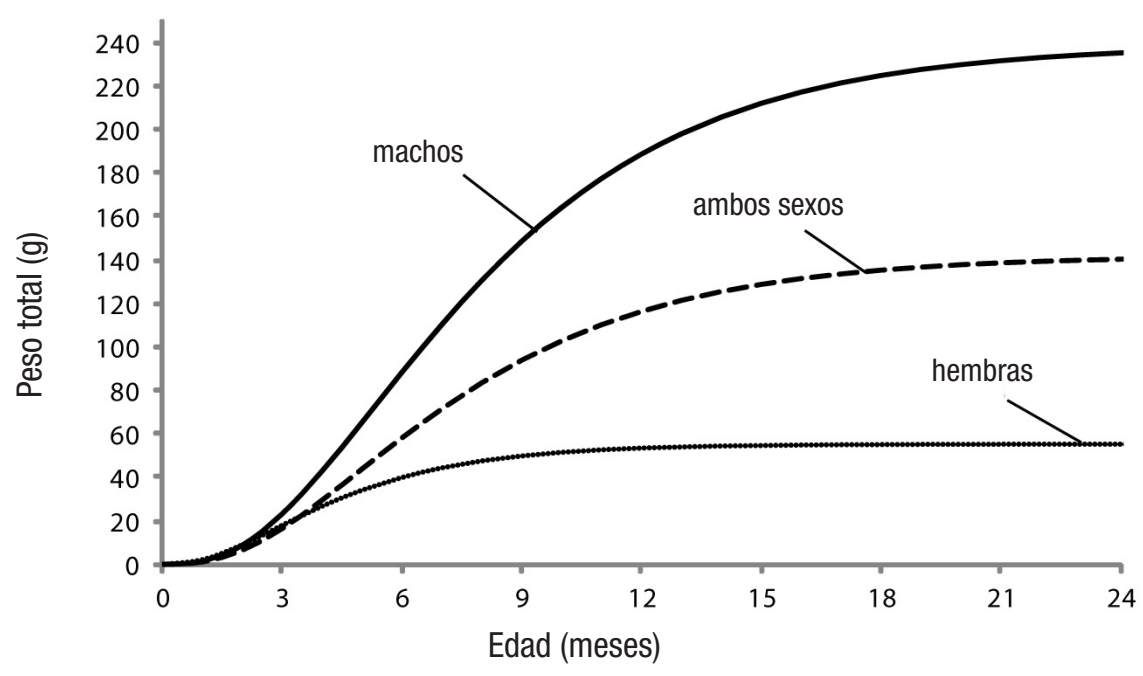

Figura 4. Curvas de crecimiento en peso de la población y de cada sexo de Macrobrachium tenellum.

características biológicas de esta especie, como son su ciclo de vida corto, rápido crecimiento, madurez temprana y alta mortalidad natural. Las tasas de mortalidad y explotación fueron congruentes entre los sexos, aunque la mortalidad y explotación fueron más altas en hembras que en machos, ya que las hembras, al tener una menor longitud y peso y una mayor abundancia, son posiblemente más vulnerables al método de pesca utilizado (atarraya), especialmente durante el periodo de reproducción (agosto a octubre), durante el cual se incrementa la actividad pesquera de este recurso. Las tasas de mortalidad natural y por pesca para ambos sexos fueron excesivamente altas; Gracia (1997) puntualiza que no es común obtener estimaciones de mortalidad natural mayor 0 igual a 0.40 mensual y que los valores superiores se consideran excesivamente altos. Sin embargo, la probabilidad de que la mortalidad natural presente variaciones es alta, ya que el valor de mortalidad natural no es estable y depende en gran medida de dife- rentes variables del ecosistema, como las variaciones estacionales y cíclicas en parámetros ambientales, las fluctuaciones en la abundancia de depredadores y las variaciones poblacionales en los patrones de distribución, agregación y migración, entre otros. La actividad pesquera se intensifica de manera importante en el periodo de reproducción, cuando se obtienen los mayores volúmenes de captura, lo que se refleja en la alta mortalidad por pesca. Las tasas de mortalidad total anual también resultaron altas, de acuerdo con los criterios de Sparre y Venema (1992), que indican que valores de $Z=0.6$ indican una explotación leve, $Z=0.9$ una explotación mediana y $Z=1.2$ una explotación intensiva. De acuerdo con este criterio, los resultados estimados en el presente estudio indican que el recurso está siendo explotado en forma intensiva. Las tasas de explotación de machos y hembras $(E=0.70$ y $\mathrm{E}=0.79$, respectivamente) indicaron que las hembras están en un nivel mayor de explotación. Si se siguen los criterios de Gulland (1971)

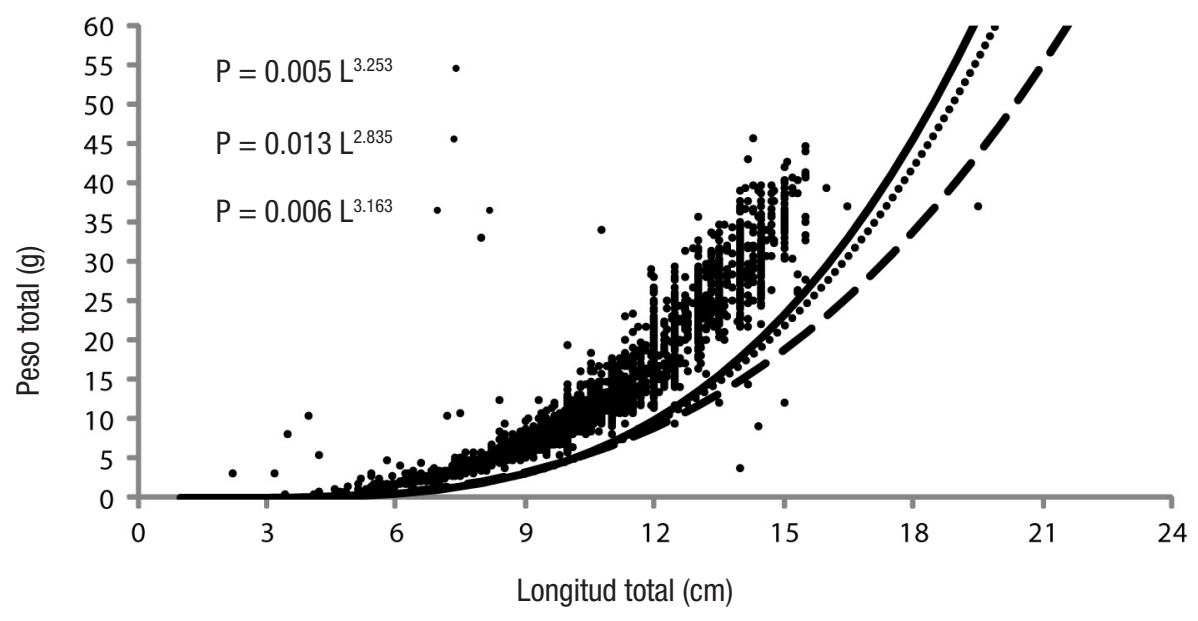

- hembras machos población

Figura 5. Relación longitud-peso de la población y de cada sexo de Macrobrachium tenellum. 
y Pauly (1983), que proponen que el valor de $E=0.5$ es un punto de equilibrio y que resultados mayores indican que el recurso está siendo sometido a una alta explotación, se puede decir que $M$. tenellum es una especie intensamente sobreexplotada en la zona de estudio. Según Pauly (1983), la sobrepesca puede tener lugar en tres formas diferentes: (i) en relación con el crecimiento poblacional, (ii) en relación con el reclutamiento del stock capturable y (iii) como sobrepesca que afecta el ecosistema. Si se siguen estos criterios, se puede sugerir que la población de camarón moya en las localidades de Mexcaltitán, San Miguel Aztlán y Boca de Camichín del río San Pedro Mezquital está sufriendo de la sobrepesca por crecimiento (abundancia). Este tipo de sobrepesca tiene lugar cuando los individuos son capturados antes de que alcancen la talla necesaria para garantizar la renovación biológica de la población. Se concluye, entonces, que el langostino Macrobrachium tenellum presenta un ciclo de vida corto, menor de dos años, una tasa de crecimiento moderada y tasas de mortalidad total y de explotación altas, que ocasionan una sobreexplotación del recurso en el área de estudio. Lo anterior hace necesario que se invierta en esfuerzos para continuar con investigaciones pesqueras que permitan evaluar y diseñar estrategias de manejo para $M$. tenellum en la pesquería del río San Pedro Mezquital, Nayarit, en una forma que sea consistente con un enfoque precautorio para la pesca responsable de este recurso.

\section{AGRADECIMIENTOS}

Se agradece a la Sociedad Cooperativa de Producción Pesquera de Mexcaltitán, San Miguel Aztlán y Boca de Camichín S. C. de R. L. de Nayarit por el apoyo en la captura de langostino moya.

\section{REFERENCIAS}

Alejo-Plata, C., J. L. Gómez-Márquez \& I. H. Salgado-Ugarte. 2011. Edad y crecimiento del dorado Coryphaena hippurus, en el Golfo de Tehuantepec, México. Revista Biología Marina y Oceanografía 46 (2): 125-134. DOI: 10.4067/S0718-19572011000200003

Arroyo-Renteria G. \& L. Magaña-Ríos. 2001. Contribución al conocimiento de las especies de Macrobrachium y Atya con especial referencia a los langostinos en el cauce del Río Baluarte (1991). Tesis de Licenciatura en Biología Pesquera, Facultad de Ciencias del Mar, Universidad Autónoma de Sinaloa, México. 58 p.

Bahamonde, N. \& I. Vila. 1971. Sinopsis sobre la biología del camarón de río del norte. Biología Pesquera, Chile 5: 3-60.

Berveton, R. J. H. \& S. J. Holt. 1957. On the dynamics of exploited fish populations. Fishery Investigation Series II, 19. London: Ministry of Agriculture, Fisheries and Food 2-19, Great Britain. 533 p.

Boschi, E. E. 1974. Biología de los crustáceos cultivables en América Latina. In: FAO (Organización para la Agricultura y la Alimentación). 1974. Actas del Simposio sobre Acuicultura en América Latina. VoIumen 2 - documentos de reseña. Informe de Pesca 159, vol. 2. Montevideo, Uruguay. $24 \mathrm{p}$.

Cabrera-Jiménez, J., M. Guzmán \& C. Kensler. 1977. Macrobrachium fishery and market in Mexico. In: Hanson, J. \& G. Goodwin (Eds.). Shrimp and prawn farming in the western hemisphere. Dowden, Hutchington and Ross, Inc. USA. pp. 315- 316
CASSIE, R. M. 1954. Some uses of probability paper in the analysis of size frequency distributions. Australian Journal of Marine and Freshwater Research 5: 513-524.

CSIRKE, J. 1980. Introducción a la dinámica de poblaciones de peces. FAO. Documento Técnico de Pesca 192: 1-82.

DOF (Diario Oficial de la Federación). 1994. Aviso por el que se da a conocer el establecimiento de épocas y zonas de veda para la pesca de diferentes especies de la fauna acuática en aguas de jurisdicción federal de los Estados Unidos Mexicanos. Diario Oficial de la Federación. México, D.F. Marzo 12: 96-103.

Espino-Barr, E., B. A. García, G. M. Puente, A. C. Zamorano, A. 0. Ahumada \& E. CaBral. 2006. Análisis preliminar de los aspectos biológicos del langostino mazacate Macrobrachium tenellum, en el estado de Colima. In: Espino-Barr, E., M. A. Carrasco \& G. M. Puente (Eds.). Memorias del III Foro Científico de Pesca Ribereña. Centro Regional de Investigaciones Pesqueras de Manzanillo, Instituto Nacional de la Pesca, SAGARPA. Jalisco, México. pp. 93-94.

Fransozo, A., F. D. Rodrigues, F. A. M. Freire \& R. C. Costa. 2004. Reproductive biology of the freshwater prawn Macrobrachium jelskii (Miers, 1877) y Macrobrachium iheringi (Ortmann, 1897) (Decapoda: Caridea: Palaemonidae) in the Botucatu region, Sao Paulo, Brasil. Nauplius 12: 119-126.

Gracia, A. 1997. Simulated and actual effects of the Brown shrimp, Penaeus aztecus, costure in Mexico. Marine Fisheries Review 59 (2): 18-24.

Gulland, J. A. 1971. Manual de Métodos para la Evaluación de Poblaciones de Peces. Ed. Acribia. Zaragoza, España. 164 p.

Guzmán, A. M., G. J. L. Rojas \& G. L. D. González. 1981. Ciclo anual de maduración y reproducción del "Chacal" Macrobrachium tenellum y su relación con factores ambientales en las lagunas costeras de Mitla y Tres Palos, Guerrero, México (Decápoda: Palaemonidae). Anales del Instituto de Ciencias del Mar y Limnología, Universidad Nacional Autónoma de México 9: 67-80.

Guzmán-Arroyo, M. 1987. Biología, ecología y pesca del langostino Macrobrachium tenellum (Smith, 1871), en lagunas costeras del estado de Guerrero, México. Tesis de Doctorado en Ciencias del Mar, Instituto de Ciencias del Mar y Limnología, Colegio de Ciencias y Humanidades, UNAM. México. 319 p.

HendRICKX, M. E. 1995. Camarones. In: Fischer, W., F. Krupp, W. Scheneider, C. Sommer, K. E. Carpenter \& V. H. Niem (Eds.). Guía FAO para la identificación de especies para los fines de pesca. Pacífico CentroOriental. FA0. Roma. pp. 417-537.

Hernández, L., G. Murugan, G. Ruiz \& A. Meada. 2007. Freshwater shrimp on the genus Macrobrachium (Decapoda Paleamonidae) from the Baja California Peninsula, México. Journal Crustaceana Biology 27 (2): 139-143.

Hernández-Sandoval, P. 2008. Efecto de la temperatura en el crecimiento y sobrevivencia del langostino Macrobrachium occidentale y del acocil Cherax quadricarinatus. Tesis de Maestría en Ciencias (Recursos Naturales y Medio Ambiente), Departamento de Acuicultura, Centro Interdisciplinario de Investigación para el Desarrollo Integral Regional, Instituto Politécnico Nacional. Sinaloa, México. 47 p. 
HolthuIs, L. B. 1952b. On a Collection of decapod crustacea from The Republic of El Salvador (Central America). Zoologische Verhandelingen 23: 1-45.

Mariappan, P. \& C. Balasundaram. 2004. Studies on the morphometry of Macrobrachium nobilii (Decapoda, Palaemonidae). Brazilian Archiv Biological and Technology 47 (3): 441-449. D0I: 10.1590/S151689132004000300015

Meruane, J. A., M. C. Morales, C. A. Galleguillos, M. A. Rivera \& H. HoSOKAWA. 2006. Experiencias y resultados de investigaciones sobre el camarón de río del norte Cryphiops caementarius (Molina, 1782) (Decapoda;Palaemonidae): historia natural y cultivo. Gayana 70 (2): 280-292. DOI: $10.4067 /$ S0717-65382006000200015

Mossolin, E. C. \& S. L. S. Bueno. 2003. Relative growth of the second pereiopod in Macrobrachium olfersi (Wiegmann, 1836) (Decapoda, Palaemonidae). Crustaceana 76: 363-376.

Musick, J. \& N. Frazer. 1999. Planificación del manejo para especies Iongevas. Contribución No. 2353 Virginia Institute of Marine Science. pp. 61-67.

New, M. B. 1988. Freshwater Prawns. Status of global aquaculture, 1987. NACA Technical Manual No. 6. A World Food Day Publication of the Network of Aquaculture Centers in Asia. Bangkok, Thailand. $58 \mathrm{p}$.

Norambuena, R. 1977. Antecedentes biológicos de Cryphiops caementarius (Molina, 1782) en el estero "El Culebrón" (Crustacea, Decapoda, Palaemonidae). Biología Pesquera, Chile 9: 7-19.

Pauly, D. 1983. Algunos métodos simples para la evaluación de Recursos pesqueros tropicales. FA0. Documento Técnico de Pesca 234: $1-52$.

Pauly, D. \& J. L. Munro. 1984. Once more on the comparison of growth in fish and invertebrates. Fishbyte 2: 21.

Pérez-Velazquez, P. A., P. Ulloa, J. L. Patiño \& J. Tovar-Ávila. 2011. La pesca del langostino (Macrobrachium tenellum) en la laguna de Mexcaltitlán, Nayarit, una alternativa económica regional. Ciencia Pesquera 19: 13-20.
RICKER, W. E. 1975. Computation and interpretation of biological statistics of fish population. Department of Fisheries and the Environment, Fisheries and Marine Service of Canada, Bulletin 191, pp. 1-382.

RIKHTER, V.A. \& V.N. EfFAnov. 1976. On one of the approaches to estimation of natural mortality of fish populations. International Commission for the Northwest Atlantic. Fisheries Research Document 76/VI/8: $1-12$.

Roman-Contreras, R. 1979. Contribución al conocimiento de la biología y ecología de Macrobrachium tenellum (Smith, 1871) (Crustacea, Decapoda, Palaemonidae). Anales del Instituto de Ciencias del Mar y Limnología, Universidad Nacional Autónoma de México 6: 137160.

Román-Contreras, R. 1991. Ecología de Macrobrachium tenellum (Decapoda: Palaemonidae) en la laguna Coyuca, Guerrero, Pacífico de México. Anales del Instituto de Ciencias del Mar y Limnología, Universidad Nacional Autónoma de México 18: 87-96.

Ruiz-Santos, H. 1988. Estudio de edad y crecimiento del langostino Macrobrachium tenellum (Smith, 1871) en la laguna de Tres Palos, Gro. Tesis de Maestría en Ciencias del Mar (Oceanografía Biológica y Pesquera), Colegio de Ciencias y Humanidades, Instituto de Ciencias del Mar y Limnología, UNAM. México. 78 p.

Signoret-Poillon, G. \& E. Soto. 1997. Comportamiento osmoregulador de Macrobrachium tenellum y Macrobrachium acanthurus (Decápoda: Palaemonidae) en diferentes salinidades. Revista Biología Tropical 45: 1085-1091.

Sparre, P. \& S. C. Venema. 1992. Introducción a la evaluación de recursos pesqueros tropicales. Parte 1- Manual. FAO. Documento Técnico de Pesca 306/2 Rev. 1. 432 p.

WALFoRd, L. A. 1946. A new graphic method of describing the growth of animals. Biological Bulletin of the Marine Biological Laboratory, Woods Hole 90:141-147.

ZaR, J. H. 1999. Biostatistical analysis. Fourth edition. Prentice Hall. EE.UU. $123 p$. 